\title{
Severe Necrotizing infection of the Perineum: Beyond Necrosectomy
}

\author{
Abdihakin M. ${ }^{1}$, Saidi $\mathbf{H}^{\mathbf{1}, \mathbf{2}}$ Affiliation: 1. Aga Khan University hospital 2. University of Nairobi. Correspondence to: Dr. Abdihakin \\ Mohammed, Aga Khan University hospital, P.0. Box 30270, 00-100, Nairobi, Kenya.
}

\section{Summary}

The evolution of systemic inflammatory response syndrome (SIRS) to septic shock is a continuum that can be stemmed using dedicated and early goal directed interventions. In the setting of necrotizing soft tissue infection, mortality approaches $100 \%$ when debridement is delayed or altogether omitted. Volume depletion, vasodilatation, myocardial depression, high metabolism and attendant global hypoxia that precede multi- organ dysfunction syndrome (MODS) and mortality need to be addressed early, avoiding delays in the emergency department, hospital ward, or the intensive care unit. Early goal directed therapy denotes the use of interventions such as administration of crystalloid solutions, vaso-active agents, blood transfusion and inotropic agents to achieve specific targets, namely, a central venous pressure of $8-12 \mathrm{mmHg}$, a mean arterial pressure of $65-90 \mathrm{mmHg}$, a urine output of $>0.5 \mathrm{mls} / \mathrm{kg} / \mathrm{hr}$, a hematocrit of $>30 \%$ and a central venous oxygen saturation of $>70 \%$ in a patient who is intubated, sedated and paralysed.We present an illustrative case of the management of severe Fournier's gangrene and how a series of misadventures at home, the A \& E and the wards contributed to the inevitable demise.

\section{Introduction}

Fournier's Gangrene is a fulminant necrotizing fascitis of the penis and scrotum (1) with reported mortality rate of $20 \%$ which peaks to $39 \%$ when debridement is delayed (2). Upon diagnosis, the disease also warrants an aggressive multimodal approach, which includes hemodynamic stabilization and broad spectrum antibiotics (3). This management strategy may assist to stem the transition to multiple organ dysfunction and mortality. Indeed, initiation of fluid resuscitation, transfusion, and vasoactive support at the A \& E reduces mortality significantly (4). This advantage is lost when the interventions are intiatied at the intensive care unit.

\section{Case}

MX was a 53 year old male who presented with five days history of pain, swelling and foul discharge of the scrotum. He had fever, nausea and anorexia but reported no history of cough, night sweats, weight loss or contact with a person with TB. His past medical history was not significant. Two weeks earlier, he used herbal medications to treat a painful leg swelling. He had a history of heavy alcohol use.

On examination, he was wasted, dehydrated and had generalized lymphadenopathy but was not pale, or jaundiced. He was hypotensive (BP 90/50 mmHg), tachycardic (pulse 137/min) and febrile (temperature $38.5^{\circ} \mathrm{C}$ ). The respiratory rate was $16 / \mathrm{min}$ and saturations were $98 \%$ on room air. Perineal findings included bilateral ischiorectal abscesses and a swollen, erythematous, crepitant scrotum with a discharging blister (Fig 1). The lower limb was excoriated, hyper-pigmented with nonpitting edema, but no signs of inflammation. Respiratory system exam revealed vesicular breath sounds bilaterally with no crepitations. The abdomen was moving with respiration and was soft and non-tender on palpation and bowel sounds were heard and normal.

MX was subsequently admitted with a diagnosis of sepsis secondary to Fournier's gangrene, resuscitated with crystalloid fluids, catheterized for output monitoring and started on ceftriaxone 1 gr twice daily, metronidazole $500 \mathrm{mg}$ three times daily and morphine infusion titrated to the pain experienced. The response to resuscitation was a blood pressure of $95 / 60 \mathrm{mmHg}$, pulse of $90 / \mathrm{min}$ and $\mathrm{SPO}_{2}$ of $98 \%$. Initial investigations showed leucocytosis (WBC-12.78 x 10 (N-92\% L-4\%) and a low platelet count $\left(84 \times 10^{9} / \mathrm{l}\right)$, hyponatraemia $\left(\mathrm{Na}^{+}-127 \mathrm{mmol} / \mathrm{l}\right)$ and elevated urea $(15.7 \mathrm{mmol} / \mathrm{l})$. The rest were normal (haemoglobin 14.5g/dl, K+-3.5mmol/l, Cr-101mmol/l, blood sugar $4.7 \mathrm{mmol} / \mathrm{l}$, urinalysis ( 2-4 WBC/HPF, no bacteria, blood+). He was HIV negative.

A scrotal ultrasound revealed a viable left testis with the 
Severe Necrotizing infection of the Perineum: Beyond Necrosectomy

Abdihakin M., Saidi H

\begin{tabular}{lllll}
\hline & & & & \\
Timing & PR(per min) & RR (per min) & BP $(\mathbf{m m H g})$ & U/O (ml/hr) \\
\hline Pre-op & 137 & $16-24$ & $95 / 50-97 / 57$ & $80-100$ \\
\hline 1st POD(HDU) & 112 & 18 & $88 / 40$ & 30 \\
\hline 1st POD (ICU) & 105 & 15 & $87 / 68$ & $15-20$ \\
\hline Post dialysis & & & $30 / 12$ & \\
\hline 2nd POD & & & $73 / 43$ & 4 \\
\hline & & & & \\
\hline Timing & PH & PCO2 (mmHg) & BE & HCO3 (mmol/L) \\
\hline Pre-op & 7.30 & 23.8 & -12.2 & 15.7 \\
\hline 1st POD(HDU) & 7.155 & 28.2 & -17.5 & 10.8 \\
\hline 1st POD (ICU) & 6.69 & 29.8 & -30.4 & 3.4 \\
\hline Post dialysis & 7.148 & 28.8 & -16.3 & 11.8 \\
\hline Normal Values & $7.35-7.45$ & $35-45$ & $-1-+1$ & $22-26$ \\
\hline
\end{tabular}

Table 1: Measurement trends for $\mathrm{MX}$

right obscured by surrounding air. Early on the second day, he was alert but clinically dehydrated. Urine output ranged between 80 and $100 \mathrm{mls} / \mathrm{hr}$. He was still tachycardic and hypotensive (table 1 ) with a respiratory rate of $24 / \mathrm{min}$ and saturation $100 \%$ on $3 \mathrm{~L}$ of oxygen. Arterial blood gas analysis showed a PH of 7.3 and base excess of -12.2 (table 1) He was taken to the operating room, induced with ketamine/midazolam and debrided under face-mask. Necrotic tissues involving the ischioanal fossae, the right and left groins, scrota and base of the penis were excised to bleeding surfaces (Figures 1 and 2) and thoroughly irrigated. Both testes were healthy.

He had a nadir blood pressure of $73 / 39 \mathrm{mmHg}$ and urine output $>30 \mathrm{mls} / \mathrm{hr}$ during the operation. He was adequately reversed, and was breathing spontaneously. A re-look surgery was planned for two days later.

Post-operatively, he was nursed in the high dependency unit. At admission to this unit, his blood pressure was $88 / 40 \mathrm{mmHg}$, RR $18 / \mathrm{min}$ and saturations $92 \%$ on $5 \mathrm{~L}$ of oxygen. Shortly after, the systolic blood pressure fell to $73 \mathrm{mmHg}$. This was attributed to the morphine infusion. One hour later however, he became unresponsive (GCS of 5/15) and developed deep sighing respirations. The CVP was $9 \mathrm{cmH}_{2} \mathrm{O}$, output $30 \mathrm{mls} /$ hour despite an input of 200mls/hr.

The arterial blood gases showed worsening acidosis (table 1). He was commenced on Norepinephrine $4 \mathrm{mcg} /$ min, Naloxone $0.2 \mathrm{mg}$, fluid boluses of haesteril and transferred to the ICU. During the initial stay in ICU he was rousable (GCS $10 / 15$ ) but remained hypotensive (BP $87 / 68 \mathrm{mmHg}$ ) with a pulse rate of $105 /$ $\mathrm{min}$, respiratory rate of $15 / \mathrm{min}$ and saturations of $100 \%$ on $4 \mathrm{~L}$ of oxygen. The groin wound discharged pus. Dopamine was added to the Norepinephrine and enteral feeding considered.

Several hours later however, he desaturated to $\mathrm{SPO}_{2} 88 \%$ and was tachypnoiec with grossly reduced air entry bilaterally. He was then intubated and mechanically ventilated on SIMV mode, PS of 16, TV of 450 , PEEP of $5, \mathrm{FiO}_{2}$ of $60 \%$ and Rate of 18. He had a low urine output of $15-20$ $\mathrm{mls} / \mathrm{hr}$. His arterial blood gases were pH-6.69 pCO $_{2}-29.8 \quad \mathrm{pO}_{2}-141 \mathrm{HCO}_{3}-3.4$ BE -30.4. He developed hypoglycemia of $2.1 \mathrm{mmols} / \mathrm{L}$ and his WBCs were $32.7 \times 10^{\circ}$ (N 92\%). His haemoglobin was $9.0 \mathrm{~g} / \mathrm{dl}$ and platelets of $42 \times 10^{9} / \mathrm{L}$.

Inotropic support was maximized and he received a stat dose of Sodium Bicarbonate. The antibiotics were changed to Imipenem 2 gr twice daily for a broader coverage as culture results were awaited and a renal review was requested for dialysis due to the severe metabolic acidosis. He was commenced on intermittent haemodialysis. Dialysis was stopped 3hrs after commencement due to a further drop in the blood pressure (30/12 $\mathrm{mmHg}$ ). He became hypothermic (temperature $34^{\circ} \mathrm{C}$ ). There was a mild improvement in the blood gasses after dialysis (table 1). MX remained hypotensive on the second postoperative day and developed generalized edema with cold and cyanosed peripheries. CVP remained $13 \mathrm{cmH}_{2} \mathrm{O}$ with urine output of $4 \mathrm{mls} / \mathrm{hr}$.

His pupils were dilated and non-reactive to light. The chest x-ray findings were consistent with ARDS. Several hours later, he went into bradycardia and did not respond to resuscitation.

\section{Discussion}

The causes of death in Fournier's gangrene include severe sepsis, coagulopathy, acute renal failure, diabetic ketoacidosis, and multiple organ failure (3). Diabetes mellitus, alcoholism, neurological deficits, malignancy, advanced age and immunosuppresion are well-known predisposing factors for Fournier's gangrene (6). 


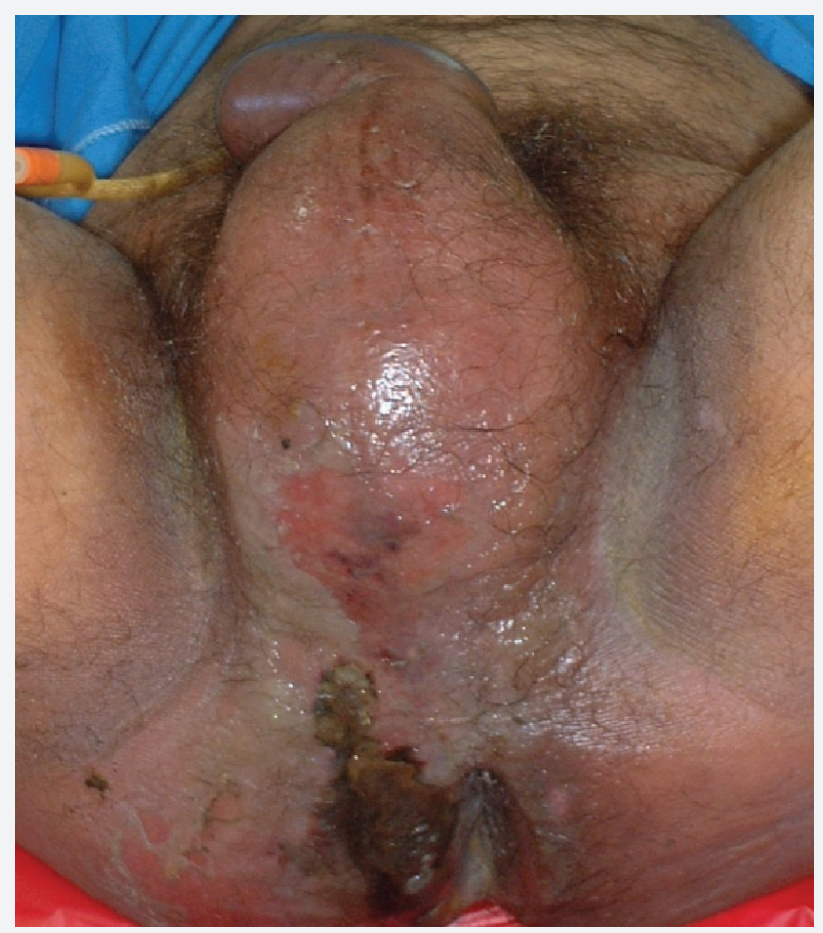

Figure 1 The scrotum is grossly swollen, erythematous with a small discharge from a blister. Note the ischiorectal abscess.

The diagnosis is mainly clinical and the application of investigations such as ultrasonography in its diagnosis is complicated $(5,7)$. Upon diagnosis, the disease warrants an aggressive multimodal approach, which includes hemodynamic stabilisation, broad spectrum antibiotics, and emergency surgical debridement (3). This article highlights the management of the case presented in the context of critical care. MX presented five days after the onset of perineal symptoms. By the time of presentation his disease had evolved from SIRS to severe sepsis. The events after admission illustrate how quickly the continuum moved to MODS and death.

Several poor prognostic factors were already at play on admission. His advanced age, heavy alcohol use and extent of the severe necrosis were poor prognostic factors. But the treatment decisions made contributed somewhat to the outcome being presented. The prevention of progression from SIRS to MODS can be optimized by the support of hemodynamics to maximize oxygen delivery, reduction of catabolism by debridement of devitalized tissue and immunological support by timely and appropriate antibiotics and a feeding strategy.

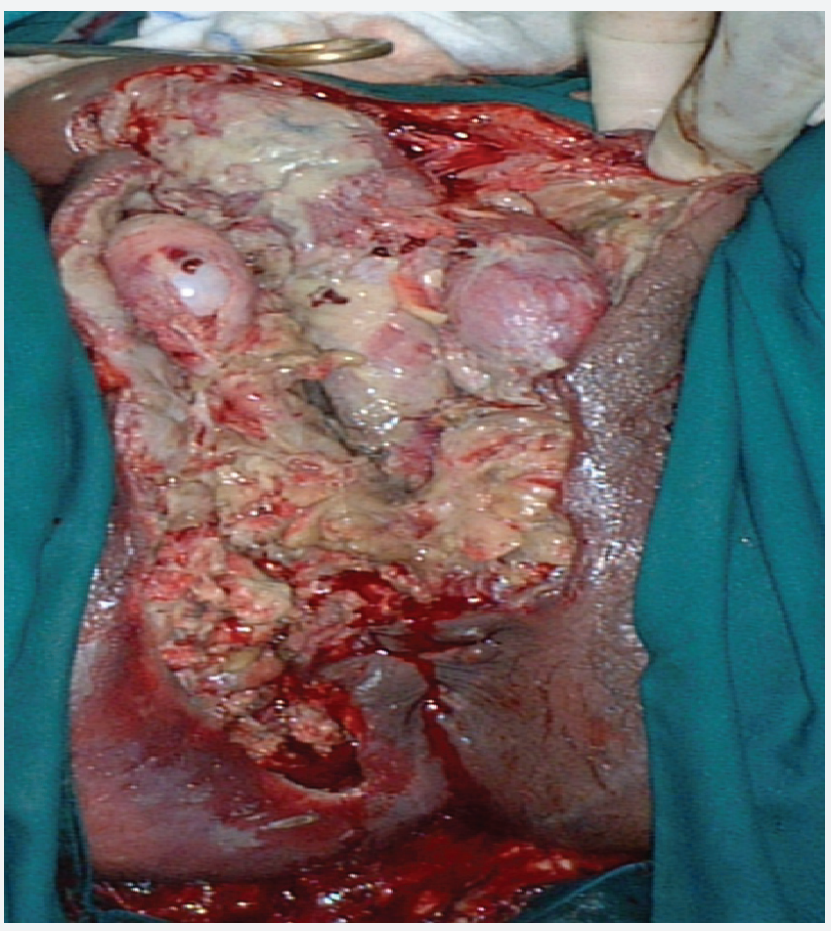

Figure 2 The scrotum and right ischioanal fossa were opened with radical debridement of necrotic tissue done. Note the healthy-looking testes.

He received prompt fluid resuscitation and broad-spectrum antibiotics as is warranted in his case. The ultrasound examination was superfluous, the ICU admission and intubation came late and the inotropic support might have been instituted earlier. There is now good level 1 evidence showing that hemodynamic resuscitation is effective when it is early and rapid. In the randomized trial by Rivers et al, initiation of fluid resuscitation, transfusion, and vasoactive support at the $\mathrm{A} \& \mathrm{E}$, all titrated to mixed venous oxygen saturation ( $\mathrm{SmvO} 2)$, reduced mortality from $46.5 \%$ to $30.5 \%$. When initiated later in the ICU, this fails to demonstrate evidence of benefit (4). Multiple surgical debridement is the rule rather than the exception in this condition, with an average of 3.5 procedures required per patient (8).

Even though the testes are classically spared in the process of Fournier's gangrene, orchidectomy, for non-viable testis, is eventually required in up to $21 \%$ patients (9). It is debatable whether surgery on the day of admission would have altered the course of events given that a period of resuscitation was needed. He did not live to get further debridements. What we failed to anticipate was 
Severe Necrotizing infection of the Perineum: Beyond Necrosectomy

Abdihakin M., Saidi H

\begin{tabular}{|c|c|c|c|c|c|c|c|c|c|}
\hline \multirow[b]{2}{*}{ Physiological Variable/Point Assignment } & \multirow[b]{2}{*}{+4} & \multicolumn{3}{|c|}{ High Abdominal Values } & \multirow{2}{*}{$\begin{array}{l}\text { Normal } \\
0\end{array}$} & \multicolumn{4}{|c|}{ Low Abdominal Values } \\
\hline & & +3 & +2 & +1 & & +1 & +2 & +3 & +4 \\
\hline Temperature (C) & More than 41 & $39-40.9$ & - & $38.5-38.9$ & $36-38.4$ & $34-35-9$ & $32-33.9$ & $30-31.9$ & Less than 29.9 \\
\hline Heart rate & More than 180 & $140-179$ & $110-139$ & - & $70-109$ & - & $56-69$ & $40-54$ & Less than 39 \\
\hline Respiration rate & More than 50 & $35-49$ & - & $25-34$ & $12-2 v 4$ & $10-11$ & $6-9$ & - & Less than 5 \\
\hline Serum sodium (mmo/L) & More than 180 & $160-179$ & $155-159$ & $150-154$ & $130-149$ & - & $120-129$ & $111-119$ & Less than 110 \\
\hline Serum potassium (mmol/L) & More than 7 & $6-6.9$ & - & $5.5-5.9$ & $3.5-5.4$ & $3-3.4$ & $2.5-2.9$ & - & Less than 2.5 \\
\hline Serum creatinine ( $\mathrm{mg} / 100 \mathrm{ml}, \mathrm{x} 2$ for acute renal failure) & More than 3.5 & $2-3.4$ & $1.5-1.9$ & - & $0.6-1.4$ & - & Less than 0.6 & - & - \\
\hline Hematocrit (\%) & More than 60 & - & $50-59.9$ & $46-49.0$ & $30-45.9$ & - & $20-29.9$ & - & Less than 20 \\
\hline White blood count (total/mm3 x 1,000) & More than 40 & - & $20-39.9$ & $15-19.9$ & $3-14.9$ & - & $1-2.9$ & - & Less than 1 \\
\hline Serum bicarbonate(venous,mmol) & More than 52 & $41-51.9$ & - & $32-40.9$ & $22-31.9$ & - & $18-21.9$ & $15-17.9$ & Less than 15 \\
\hline
\end{tabular}

Table 2: Founier's Gangrene severity Index $(11,12)$

Begin resuscitation immediately in patients with hypotention or elevated serum lactate of greater than $4 \mathrm{mmol} / \mathrm{L}$,using either crystalloids or colloids. Give fluid challenges of $1,000 \mathrm{~mL}$ of crystalloids or 300 to $500 \mathrm{~mL}$ of colloids over the course of 30 minutes. More rapid and larger volumes may be required in sepsis-induced tissue hypoperfusion.

\section{Resuscitation goals include the following:}

- Central venous pressure (CVP) of 8 to $12 \mathrm{~mm} \mathrm{Hg}$ is recommended in the presence of mechanical ventilation or pre-existing decreased ventricular compliance.

- Mean arterial pressure of greater than or equal to $65 \mathrm{~mm} \mathrm{Hg}$

- Urine output of greater than or equal to $0.5 \mathrm{~mL} / \mathrm{kg}$ per hour

- Central venous (superior vena cava) oxygen saturation $\left(\mathrm{ScvO}_{2}\right)$ of graeter than or equal to $70 \%$ or mixed venous oxygen saturation $\left(\mathrm{ScvO}_{2}\right)$ of greater than or equal to $30 \%$ and/or start dobutamine infusion.

If venous $\mathrm{O}_{2}$ saturation target is not achieved, consider further fluid,transfuse packed red blood cells if required to hematocrit of greater than or equal to $30 \%$, and/or start dobutamine infusion.

Table 3: The surviving sepsis campaign protocol for the initial haemodynamic resuscitation in severe sepsis and septic shock (15)

that surgery of devitalized tissues would release plenty of mediator factors which would vasodilate the system and lead to hemodynamic alterations and ARDS as witnessed during his HDU and ICU stays. MX's admission to the ward and later HDU increased the chances of misadventures that would propagate the evolution to MODS. His neurological deterioration was attributed to opioid effects yet CNS dysfunction occurs in over $70 \%$ of critically ill patients (10) and is a component of MODS scoring. Although he was finally transferred to the ICU, he was not intubated immediately.

ICU protocols shown to critically impact on septic patients including low tidal volume ventilation, tight glycemic control and enteral feeding were not given a chance to arrest the evolution of sepsis to MODS in this patient. Moreover, the mode of dialysis, i.e. intermittent haemodialysis may have been counterproductive, but with the lack of facilities for continuous arteriovenous haemofiltration, which has a much less risk of inducing hypotension (14), intermittent haemodialysis might have been the only available option. The cumulative events that occurred within the two days of hospital stay may be predictive of the ultimate outcome.

The prognostication system (Fournier's gangrene severity index) by Laor et al (11) is based on clinical and laboratory parameters (table2), much the same as the APACHE scoring system used in the critical care unit. With this index, any deviation from homeostasis is associated with a worse prognosis. A severity index score threshold of 9 is associated with a $75 \%$ probability of survival according to Laor et al (11). In one recent study, a 96\% survival rate was reported for patients with a score $<9$ while a $46 \%$ mortality rate was recorded for those with a Fournier's gangrene severity index of 9 or greater (sensitivity $71.4 \%$, specificity 90\%) (12).

Our case had a severity index of 8 at admission and thus had a fairly good prognosis. Although MODS can sometimes evolve and worsen despite optimal care, the critical care issues highlighted in this article contributed significantly to his mortality. 


\section{Conclusion}

Necrotizing soft tissue infection can rapidly evolve to multiple organ dysfunction if not recognized early enough and emergent surgical debridement instituted. The recognition that septic shock is the most common cause of death in these patients and prompt intensive care monitoring and urgent correction of clinical syndromes as they develop sequentially could be life saving. Early goal directed therapy for severe sepsis and septic shock has been recognized as critical and in a recent venture by a team of international experts under the auspices of the Surviving Sepsis Campaign, management guidelines for severe sepsis and septic shock were developed following the original study by Rivers (Table 3) (13). Immediate institution of evidence based critical care principles such as the early goal directed therapy plays an important role in the recovery from this disease and the case highlighted here is an example of how improper recognition of the severity of the disease and delayed institution of evidence-based appropriate critical care measures can have devastating consequences.

\section{Acknowledgement}

Presented at the Surgical Society of Kenya Conference at PanAfric Hotel in May 2008.

\section{References}

1. Fournier J.A. Gangrene foudroyante de la verge. Med Pract 1883;4: 589-97.

2. Korkut M, Icoz G et al. Outcome analysis in patients with
Fournier's gangrene. Report of 45 cases. Dis Col Rectum 2003;46:649-52.

3. Thwaini A., Khan A., Malik A. et al. Fournier's gangrene and its emergency management. Postgrad Med J 2006;82:516519

4. Rivers E., Nguyen B., Havstad S, et al. Early goal directed therapy in the treatment of severe sepsis and septic shock. $\mathrm{N}$ Engl. J. Med; 2001; 345: 1368-1377

5. Eke N. Fournier's gangrene: a review of 1726 cases BJS 2000;87: 718-728

6. Ayhan V, Ferda V. Fournier's gangrene: the development of a classical pathology. BJU Int 2007;99:1218-20.

7. Raul N., Uppot, Howard M, levy et al. Fournier Gangrene Radiology 2003; 226:115-117

8. Chawla SN, Gallop C, Mydlo JH. Fournier's gangrene: an analysis of repeated surgical debridement. Eur Urol 2003;43:572-5

9. Benizri E, Fabiani P, Migliori G, et al. Gangrene of the perineum. Urology 1996;47:935-9.

10. Razvi SM, Bone I Neurological consultations in the medical intensive care unit. J Neurol, Neurosur., psych, 2003; 74(S3): $16-23$

11. Laor E et al. Outcome prediction in patients with Fournier's gangrene. J Urol 1995;154: 89-92.

12. Corcoran Corcoran AT, Smaldone MC, Gibbons EP Validation of the Fournier's Gangrene Severity Index in a Large Contemporary Series J Urol 2008; 180: 944 - 948.

13. Dellinger RP, Levy MM, Carlet JM, Bion J, et al.: Surviving Sepsis Campaign: international guidelines for management of severe sepsis and septic shock: 2008. Intensive Care Med 2008, 34:17-60. 